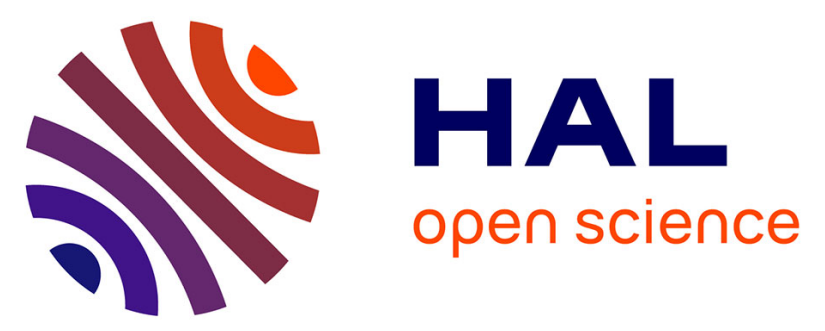

\title{
Synthesis of heteroarenes dyads from heteroarenes and heteroarylsulfonyl chlorides via Pd-catalyzed desulfitative $\mathrm{C}-\mathrm{H}$ bond heteroarylations
}

Besma Saoudi, Adbelmadjid Debache, Jean-François Soulé, Henri Doucet

\section{- To cite this version:}

Besma Saoudi, Adbelmadjid Debache, Jean-François Soulé, Henri Doucet. Synthesis of heteroarenes dyads from heteroarenes and heteroarylsulfonyl chlorides via $\mathrm{Pd}$-catalyzed desulfitative $\mathrm{C}-\mathrm{H}$ bond heteroarylations. RSC Advances, 2015, 5 (80), pp.65175-65183. 10.1039/C5RA07762A . hal-01188230

HAL Id: hal-01188230

https://hal-univ-rennes1.archives-ouvertes.fr/hal-01188230

Submitted on 17 Sep 2015

HAL is a multi-disciplinary open access archive for the deposit and dissemination of scientific research documents, whether they are published or not. The documents may come from teaching and research institutions in France or abroad, or from public or private research centers.
L'archive ouverte pluridisciplinaire HAL, est destinée au dépôt et à la diffusion de documents scientifiques de niveau recherche, publiés ou non, émanant des établissements d'enseignement et de recherche français ou étrangers, des laboratoires publics ou privés. 


\section{Synthesis of Heteroarenes Dyads from Heteroarenes and Heteroarylsulfonyl Chlorides via Pd-Catalyzed Desulfitative $\mathbf{C}-\mathbf{H}$ bond Heteroarylations}

Besma Saoudi, a,b Adbelmadjid Debache, *b Jean-François Soulé a and Henri Doucet a*

a Institut des Sciences Chimiques de Rennes, UMR 6226 CNRS-Université de Rennes 1 "Organométalliques, Matériaux et Catalyse", Campus de Beaulieu, 35042 Rennes, France

${ }^{b}$ Laboratoire de synthèse des molecules à intérêt biologique Université Mentouri-Constantine, 25000 Constantine, Algeria

Abstract: We report herein on the palladium-catalyzed direct heteroarylation of heteroarenes (e.g., pyrroles, furans, and thiophenes) in which heteroarylsulfonyl chlorides are used as coupling partners through a desulfitative cross-coupling. These C-H bond functionalizations occurred at the $\alpha$-position in the case of pyrrole and furan derivatives, while in the case of thiophenes the $\mathrm{C}-\mathrm{H}$ bonds at $\beta$ position have been heteroarylated. This methodology represents a very simple access to heteroaryl dyads. Moreover, some examples of heteroaryl triads have been synthetized via iterative $\mathrm{C}-\mathrm{H}$ bond arylations.

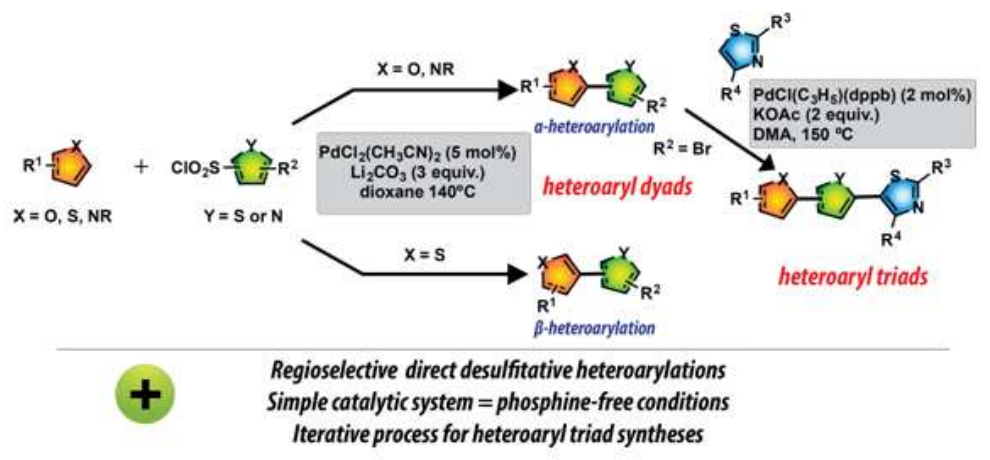

\section{Introduction}

Polyheteroaromatic compounds are well represented in organic chemistry. This motif is present in many pharmaceuticals. For examples, RITA an heteroaryl triads containing a furan 2,5 -substituted by thiophenes, is a potent multi-target drug against cancer. ${ }^{1}$ ER-38930, which possesses a pyrazole core, was also shown to markedly activate transactivation of the RARR receptor. ${ }^{2}$ Benzofuranyl-pyrrole $^{2}$ derivative $\mathbf{I}$ act as potent retinoic acid receptor $\alpha$ agonist. ${ }^{3}$ Prodigiosine is a bright red tripyrrole pigment isolated from Serratia marcescens, which displays several biological activities such as antibacterial, anticoccidial, antimalarial, and antifungal activities, and is often used as a biochemical tool. Polyheteroaromatics can be also found in organometallic chemistry as ligands (e.g., bipyridines, polythiophenes, ...) and for the preparation of electronic devices.

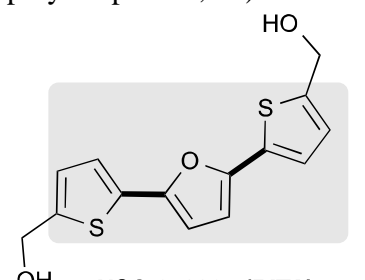

OH NSC 652287 (RITA)

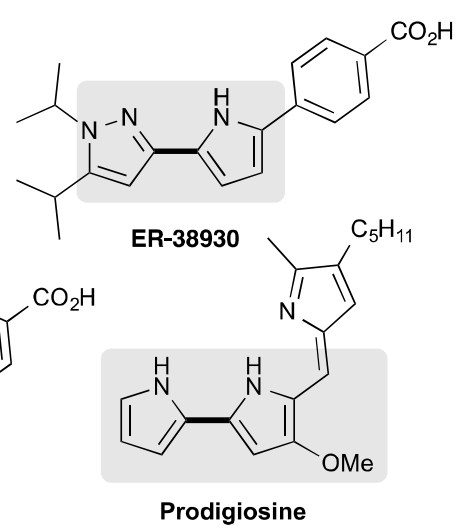

Figure 1. Relevant bioactive polyheteroaryl compounds 
The abundance of polyheteroaromatic structures in organic compounds led to intense efforts to develop new methods for their synthesis over the last few decades. ${ }^{4}$ Palladium catalysis is a very useful tool for the $\mathrm{C}-\mathrm{C}$ bond formation. ${ }^{5}$ The synthesis of heteroaryl dyads has been widely reported using palladium catalysis from organometallic reagents with heteroaryl halides (Figure 2A). Major contributions focused on the synthesis of C2-C2' heteroaryl dyads - which found some applications as electronic devices- ${ }^{6}$ but a few examples of the synthesis C3-C2' or C3-C3' heteroaryl dyads have been also reported using trialkyl(heteroaryl)stannane derivatives and 2haloheteroaryls, ${ }^{7}$ or 3-haloheteroaryls. ${ }^{7 \mathrm{~b}, 8}$ On the other hand, palladium-catalyzed direct $\mathrm{C}-\mathrm{H}$ bond arylation has emerged as one of the most suitable and eco-friendly alternative for the formation of $\mathrm{C}-\mathrm{C}$ bonds, because of a simpler access to reactants with generation of lower amount of waste. ${ }^{9}$ Palladium-catalyzed direct arylation of heteroarenes with heteroaryl halides for the formation of C2-C2' or C2C3' heteroaryl dyads has been reported, ${ }^{10}$ which includes intramolecular reactions, ${ }^{11}$ moreover some applications in the synthesis of electronic devices were also described (Figure 1B). ${ }^{12}$

More recently, we reported the use of benzenesulfonyl chlorides as an original alternative to aryl halides for the direct arylation of heteroarenes through palladium-catalyzed desulfitative $\mathrm{C}-\mathrm{H}$ bond functionalizations. ${ }^{13}$ Benzenesulfonyl chlorides exhibits several advantages such as commercial availability for many of them at an affordable cost including heteroarylsulfonyl chlorides. They can also be easily prepared from sulfonic acids or sulfur substrates by chlorination. ${ }^{14}$ In addition, palladium-catalyzed direct arylation of thiophenes with benzenesulfonyl chlorides offered different regioisomers than the reaction performed with aryl bromides. ${ }^{13 a}$ In the course of our previous study on Pd-catalyzed desulfitative cross-coupling reactions, we demonstrated on one example that methyl 3(chlorosulfonyl)thiophene-2-carboxylate might be used as coupling partner to afford a 3-3' dithiophene derivative in good yield (Figure 1C). To the best of our knowledge, this is the only example of such desulfitative direct heteroarylation reported in the literature. As a wide scope of heteroarylsulfonyl chlorides are commerically available and due to the lack of information concerning their reactivities in palladium-catalyzed desulfitative cross-coupling, their potentials in the synthesis of heteroaryl dyads and triads needed to be studied (Figure 1D).

\section{A. Pd-catalyzed heteroarylations using organometallic reagents}

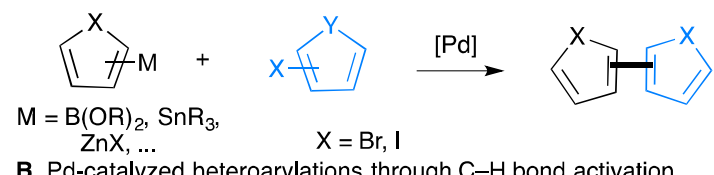

B. Pd-catalyzed heteroarylations through $\mathrm{C}-\mathrm{H}$ bond activation

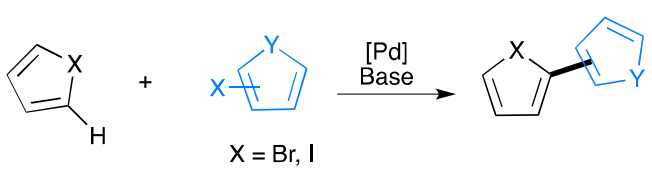

C. Pd-catalyzed desulfitative heteroarylation through $\mathrm{C}-\mathrm{H}$ bond activation

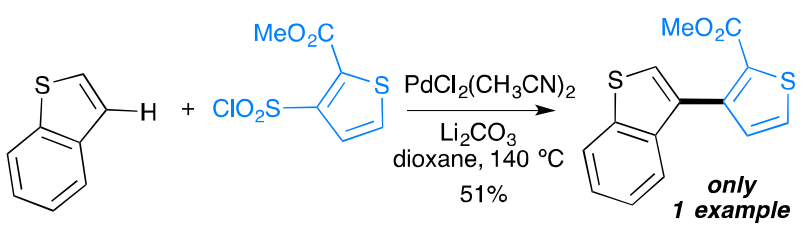

D. Generalization of Pd-catalyzed desulfitative heteroarylation (this work)

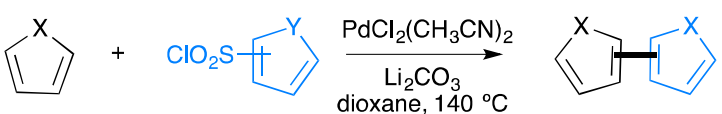

Figure 2. Palladium-catalyzed heteroarylation reactions

\section{Result and Discussion}

Firstly, we decided to investigate the reactivity of thiophene-3-sulfonyl chloride in palladium catalyzed desulfitative arylation reaction of 5-membered rings containing one heteroelement. We selected a fully substituted thiophene-3-sulfonyl chloride to prevent homocoupling side reactions. We attempted the heteroarylation of 1-methylpyrrole with 4-bromo-2,5-dichlorothiophene-3-sulfonyl chloride as heteroarylating agent using our previous best reaction conditions for such desulfitative $\mathrm{C}-\mathrm{H}$ bond arylations, ${ }^{13 \mathrm{c}}$ namely $5 \mathrm{~mol} \%$ $\mathrm{PdCl}_{2}\left(\mathrm{CH}_{3} \mathrm{CN}\right)_{2}$ in the presence of 3 equiv. of lithium carbonate in dioxane at $140{ }^{\circ} \mathrm{C}$. We found that the reaction proceeded smoothly to afford the 2-heteroarylation product $\mathbf{1}$ in $81 \%$ yield. It is important to note that all $\mathrm{C}$-halogen bonds including the $\mathrm{C}-\mathrm{Br}$ one were untouched during the reaction, which allows further transformations. Then, we attempted the reaction using furan derivatives as the coupling partners. Both 2- $n$-butylfuran and menthofuran were regioselectively heteroarylated at C5-position to give $\mathbf{2}$ and $\mathbf{3}$ in $64 \%$ and $70 \%$ yields, respectively. Interestingly, the reaction conditions tolerates enolizable ketone substituent as 1-(furan-2-yl)propan-2-one reacted with 4-bromo-2,5-dichlorothiophene-3-sulfonyl chloride to provide the desired arylated product 4 in $65 \%$ yield. The reaction 
performed with benzofuran as the starting material led to the unexpected debrominated coupling product $\mathbf{5}$ in $42 \%$ yield. Benzothiophene was arylated at C3-position to give the desired thiophene dyad $\mathbf{6}$ in moderate yield. Nevertheless, this result could be an opening wedge in original and efficient synthesis of 3,3'-bithiophene derivatives. We investigated next the reactivity of other thiophene3 -sulfonyl chlorides. Thiophene bearing only chloro or methyl substituents at the positions 2 and 5 afforded the coupling products with 1-methylpyrrole 7 and 9 in lower yields of 51\% and 36\%, respectively. Moreover, no reaction occurred between 2,5-dichlorothiophene3 -sulfonyl chloride and 2-n-butylfuran. Finally, we found that the reaction proceeded in high yield with a thiophene-3-sulfonyl chloride bearing an electron-withdrawing group at $\mathrm{C} 2$. Indeed, the desulfitative heteroarylation in which methyl 3-(chlorosulfonyl)thiophene-2carboxylate was used as coupling partner allowed the formation of the desired products 10-14 in good yields whatever the heteroarene.
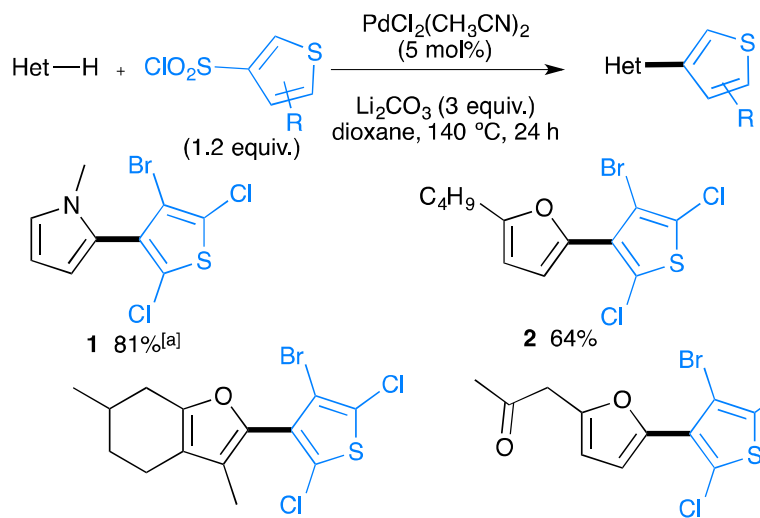
derivatives $\mathbf{1 7}$ and $\mathbf{1 8}$ in 55\% and 48\% yields, respectively. Again, these reaction conditions were completely chemoselective, as C-X bond was not involved in the direct arylation process, allowing further transformations through iterative processes. 1-Benzylpyrrole has been heteroarylated affording $\mathbf{1 9}$ in $62 \%$ yield using the most reactive 5-chlorothiophene-2-sulfonyl chloride; whereas, 2- $n$-butylfuran was found to be unreactive. 3-Heteroarylated 1,2-dimethylindole 21 was synthetized in moderate yield using 2-thiophene-2-sulfonyl chloride.

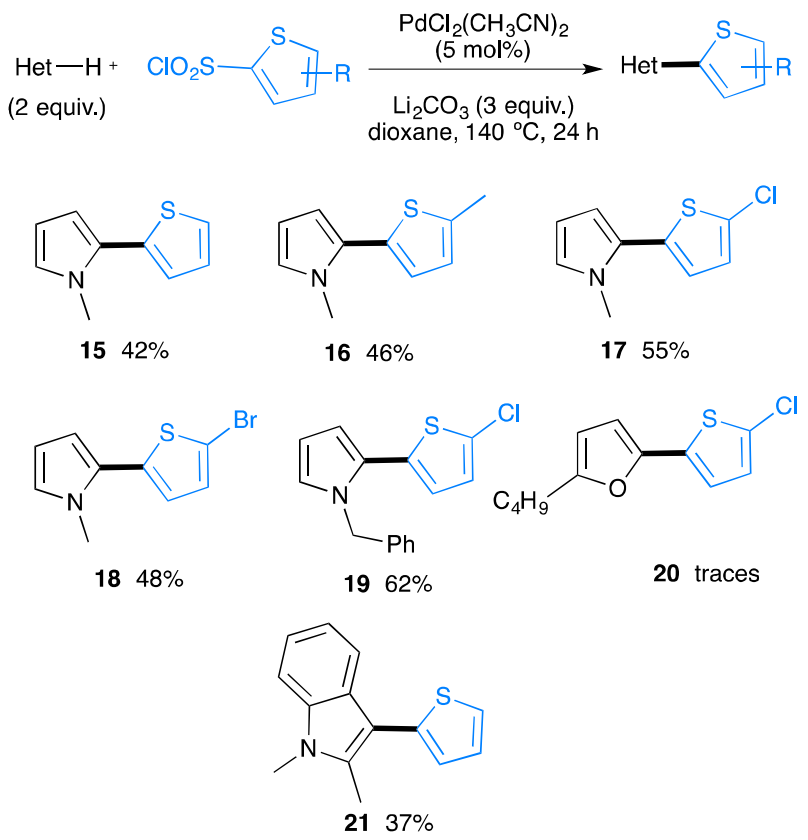

Scheme 2. Palladium-catalyzed direct desulfitative heteroarylations using thiophene-2-sulfonyl chloride derivatives.

Next, we examinated the reactivity of nitrogen containing heteroarylsulfonyl chloride derivatives as the heteroarylating agents (Scheme 3). Pyridine-3-sulfonyl chloride displayed a very low reactivity. For example, the coupling products with 1-methylpyrrole or 2-nbutylfuran gave $\mathbf{2 2}$ and $\mathbf{2 3}$ in only $31 \%$ and $12 \%$ yields, respectively. This heteroarylpyridine synthesis pathway is not attractive compared to the reaction performed using 3-bromopyridine as the arylation agent through also a $\mathrm{C}-\mathrm{H}$ bond activation. ${ }^{15}$ On the other hand, the preparation of 2-bromo-1-methylpyrrole is challenging due to over-bromination or decomposition, ${ }^{16}$ whereas 1 -methylpyrrole2-sulfonyl chlorides can be easily prepared from 1-methylpyrroles by chlorosulfonylation using chlorosulfuric acid. Moreover, some pyrrole-2-sulfonyl chlorides are commercially available. The reaction between 1-methylpyrrole and methyl 5-(chlorosulfonyl)-1methylpyrrole-2-carboxylate allowed the formation of pyrrole dyad $\mathbf{2 4}$ in $69 \%$ yield.

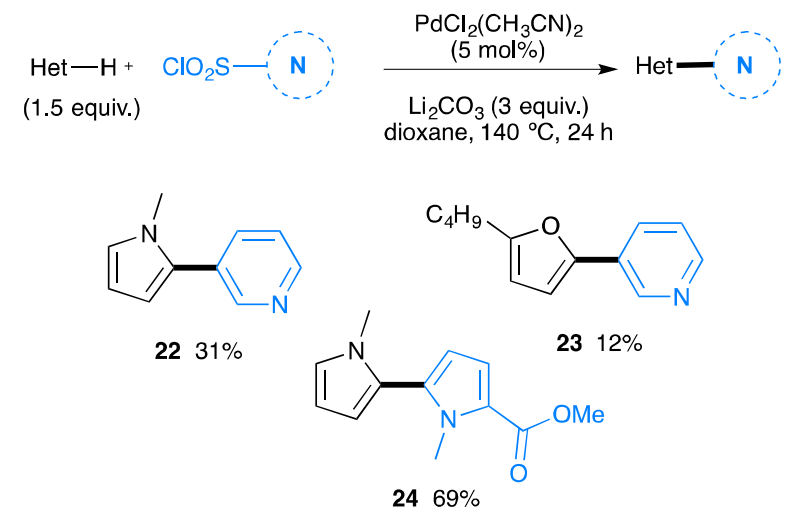

Scheme 3. Palladium-catalyzed direct desulfitative heteroarylations using nitrogen-containing sulfonyl chloride derivatives.

In order to get a wider substrate scope, the synthesis of a pyrazole unit bearing a chlorosulfonyl function was attempted. Starting from 3methyl-1-phenylpyrazole, we expected that the use of chlorosulfonic acid would provide the pyrazole $\mathbf{2 5}$, in which the chlorosulfonyl function would be introduced at the C5-position. However, we only observed the formation of the regioisomer 26, which is formed by the activation of the para $\mathrm{C}-\mathrm{H}$ bond of the phenyl ring (Scheme 4, top). This regioselectivity is different to the bromination of pyrazole with NBS, which proceeds at the pyrazole C4-position. With substrate $\mathbf{2 6}$ in hands, we investigated its reactivity in palladium-catalyzed desulfitative arylations for the synthesis of (hetero)aryl triads (Scheme 4, bottom). The arylation of 1-methylpyrrole took place at the 
expected $\mathrm{C} 2$ position allowing the formation of the triaryl $\mathbf{2 7}$ in $72 \%$ yield, while 2-methylthiophene was arylated at the C4-position to give the desired product $\mathbf{2 8}$ in $63 \%$ yield. It is important to note that such triaryls exhibit a similar structure to N6022, which is a potent $S$-nitrosoglutathione reductase inhibitor in clinical development $-{ }^{17}$ and this elegant synthetic pathway might provide an easier access to new analogues.

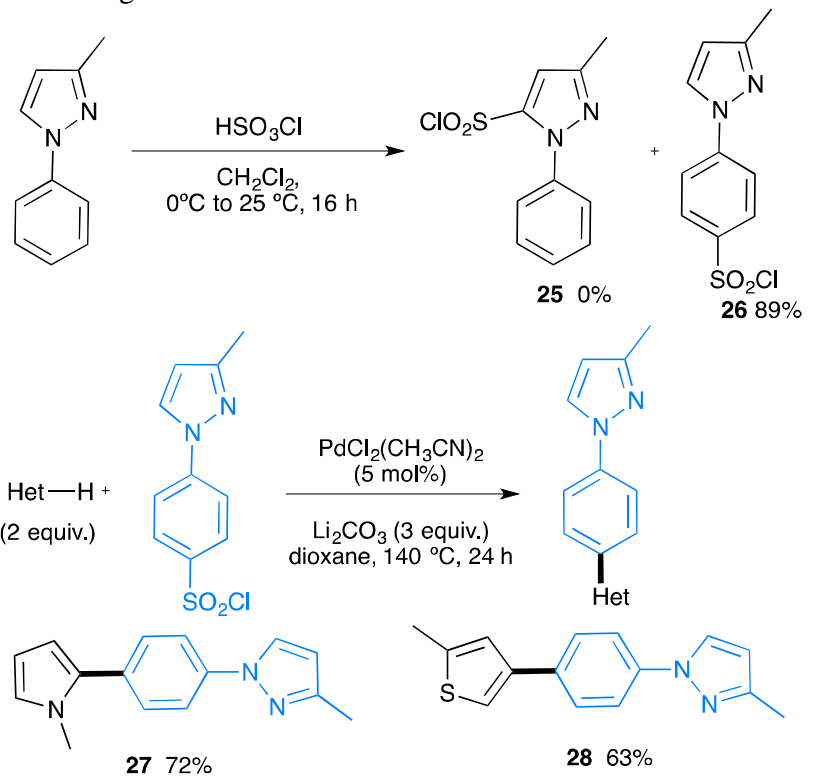

Scheme 4. Synthesis of (hetero)aryl triads from 4-(3-methylpyrazol-1-yl)benzenesulfonyl chloride via Pd-catalyzed direct desulfitative arylation.

Finally, thanks to the high chemoselectivity of these palladium-catalyzed desulfitative heteroarylations, in which the C-Br bonds remained untouched, we conducted a second direct arylation for the synthesis of heteroaryl triad compounds (Scheme 5). As examples, the derivative 18, which bears a $\mathrm{C}-\mathrm{Br}$ bond at $\mathrm{C} 2$-position on the thiophene ring, was heteroarylated -using our previous reaction conditions- ${ }^{10 \mathrm{i}}$ affording the triad $\mathbf{2 9}$ and $\mathbf{3 0}$ in $\mathbf{7 8 \%}$ and $82 \%$ yields, respectively, through a $\mathrm{C}-\mathrm{H}$ bond activation of thiazoles.

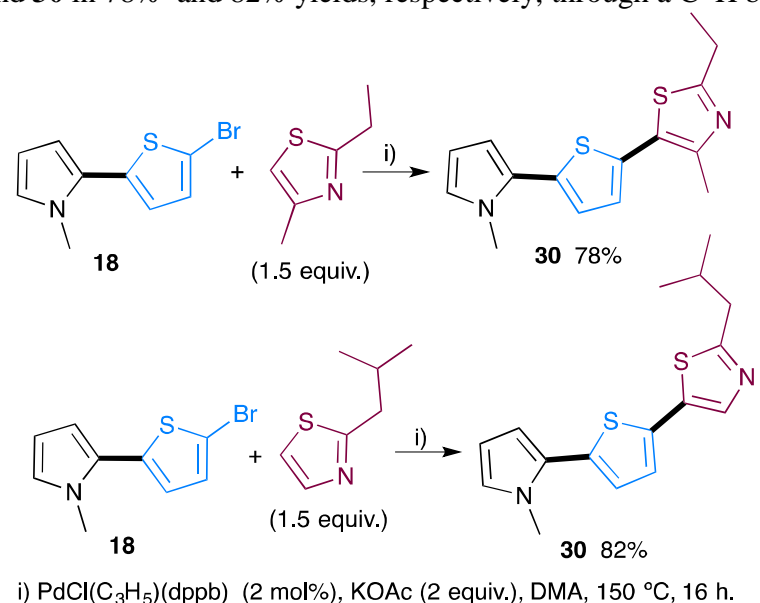

Scheme 5. Application to the synthesis of heteroaryl triads.

As heteroaryl dyads are important building blocks in the preparation of pharmaceuticals or electronic devices, we demonstrated the scalability of Pd-catalyzed desulfitative heteroarylation. The reaction was conducted on $10 \mathrm{mmol}$ scale and afforded $2.5 \mathrm{~g}$ of the compound 3 after purification (Scheme 6).

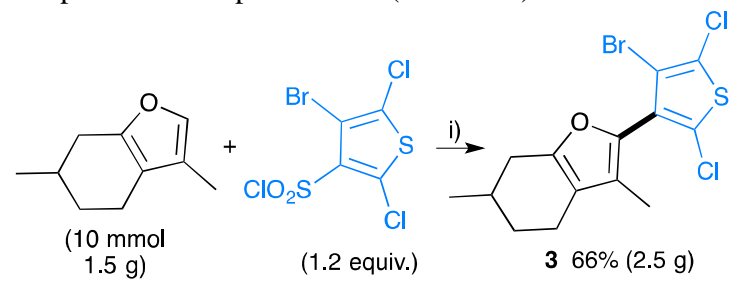

i) $\mathrm{PdCl}_{2}\left(\mathrm{CH}_{3} \mathrm{CN}\right)_{2}(5 \mathrm{~mol} \%), \mathrm{Li}_{2} \mathrm{CO}_{3}$ (3 equiv.), 1,4-dioxane, $140{ }^{\circ} \mathrm{C}, 24 \mathrm{~h}$

Scheme 6. Application to gram-scale synthesis. 
Although the exact mechanism is not completely elucidated, we can propose a reasonable pathway for palladium-catalyzed direct heteroarylation of heteroarenes (Figure 3). Based on Dong and co-workers report, ${ }^{18}$ the first step might be an oxidative addition of the heteroarylsulfonyl chloride to $\mathrm{Pd}(\mathrm{II})$ to afford the $\mathrm{Pd}(\mathrm{IV})$ intermediate $\mathbf{A}$. Then, a $\mathrm{SO}_{2}$ extrusion occurred to afford electrophilic arylpalladium(II) specie B. An electrophilic palladation of thiophene at $\beta$-position affords the intermediate $\mathbf{C}$; whereas with pyrroles and furans, the electrophilic palladation occurred at $\alpha$-position affording $\mathbf{E}$ intermediate. Then, the base assisted the rearomatization process gives the intermediates $\mathbf{D}$ and $\mathbf{F}$, which undergo reductive elimination to afford the desired arylated products and regenerates the Pd(II) catalysts. However, a Pd(0)/P(II) catalytic cycle cannot be excluded.

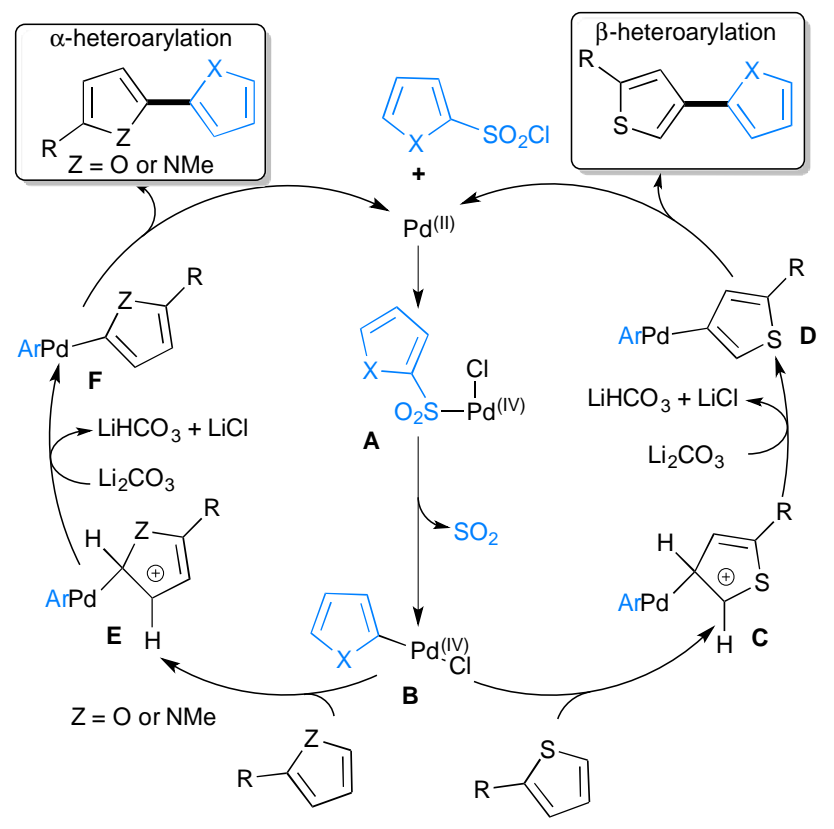

Figure 3. Proposed mechanism for palladium-catalyzed desulfitative heteroarylation reactions.

\section{Conclusions}

In summary, we have developed a new methodology for the one-step synthesis of heteroaryl dyads based on palladium-catalyzed desulfitative heteroarylation through a $\mathrm{C}-\mathrm{H}$ bond activation of heteroarenes. We established that $5 \mathrm{~mol} \% \mathrm{PdCl}_{2}\left(\mathrm{CH}_{3} \mathrm{CN}\right)_{2}$ catalyst in the presence of $\mathrm{Li}_{2} \mathrm{CO}_{3}$ as base in dioxane promotes the desulfitative heteroarylation of a variety of heteroarenes such as thiophenes, furans, and pyrroles -which is the most reactive substrates- with diverse heteroarylsulfonyl chlorides as coupling partners. Thiophene-3sulfonyl chloride derivatives generally displayed a high reactivity, while thiophene-2-sulfonyl chloride derivatives have been smoothly reacted only with 1-methylpyrrole. Other heteroarylsulfonyl chlorides containing nitrogen atom have also been successfully used. It is important to note that with heteroarylsulfonyl chlorides, uncommon functionalization at the C3-position occured with thiophenes. Moreover, other regioisomers could be obtained using chlorosulfonylation instead of bromination, as demonstrated with a 1phenylpyrazole. In addition, such cross-coupling reactions are very chemoselective as $\mathrm{C}-\mathrm{Br}$ bonds were untouched allowing iterative direct arylations for the synthesis of heteroaryl triads in only two steps.

\section{Experimental section}

General: All reactions were carried out under argon atmosphere with standard Schlenk-tube techniques. HPLC grade 1,4-dioxane was used and stored under argon without further purification. ${ }^{1} \mathrm{H}$ NMR spectra were recorded on Bruker GPX (400 MHz or $300 \mathrm{MHz}$ ) spectrometer. Chemical shifts (d) were reported in parts per million relative to residual chloroform $\left(7.26 \mathrm{ppm}\right.$ for ${ }^{1} \mathrm{H} ; 77.0 \mathrm{ppm}$ for $\left.{ }^{13} \mathrm{C}\right)$, constants were reported in Hertz. ${ }^{1} \mathrm{H}$ NMR assignment abbreviations were the following: singlet (s), doublet (d), triplet (t), quartet (q), doublet of doublets (dd), doublet of triplets (dt), and multiplet (m). ${ }^{13} \mathrm{C}$ NMR spectra were recorded at $100 \mathrm{MHz}$ on the same spectrometer and reported in ppm. All reagents were weighed and handled in air.

Preparation of the $\mathbf{P d C l}\left(\mathbf{C}_{3} \mathbf{H}_{5}\right)(\mathbf{d p p b})$ catalyst: ${ }^{19}$ An oven-dried $40 \mathrm{~mL}$ Schlenk tube equipped with a magnetic stirring bar under argon atmosphere, was charged with $\left[\mathrm{Pd}\left(\mathrm{C}_{3} \mathrm{H}_{5}\right) \mathrm{Cl}_{2}(182 \mathrm{mg}, 0.5 \mathrm{mmol})\right.$ and dppb $(426 \mathrm{mg}, 1 \mathrm{mmol}) .10 \mathrm{~mL}$ of anhydrous dichloromethane were added, then, the solution was stirred at room temperature for twenty minutes. The solvent was removed in vacuum. The powder was used without purification. $\left({ }^{31} \mathrm{P} 381 \mathrm{MHz}, \mathrm{CDCl}_{3}\right) \delta$ $=19.3(\mathrm{~s})$.

General procedure for synthesis of heteroarylated heteroarenes: To a $25 \mathrm{~mL}$ oven dried Schlenk tube, heteroarylsulfonyl chloride (1-1.2 mmol), heteroarenes (1-4 mmol), $\mathrm{Li}_{2} \mathrm{CO}_{3}(0.222 \mathrm{~g}, 3 \mathrm{mmol}), 1$,4-dioxane $(2 \mathrm{~mL})$ and $\mathrm{PdCl}_{2}\left(\mathrm{CH}_{3} \mathrm{CN}\right)_{2}(12.9 \mathrm{mg}, 0.05 \mathrm{mmol})$ were successively added. The 
reaction mixture was evacuated by vacuum-argon cycles ( 5 times) and stirred at $140{ }^{\circ} \mathrm{C}$ (oil bath temperature) for $24 \mathrm{~h}$ (see tables and schemes). After cooling the reaction at room temperature and concentration, the crude mixture was purified by silica column chromatography to afford the heteroarylated heteroarens.

2-(4-Bromo-2,5-dichlorothiophen-3-yl)-1-methylpyrrole (1): 1-Methylpyrrole (0.162 g, 2 mmol) and 4-bromo-2,5-dichlorothiophene-3-sulfonyl chloride $(0.330 \mathrm{~g}, 1 \mathrm{mmol})$ affords 1 in $81 \%(0.252 \mathrm{~g})$ yield.

${ }^{1} \mathrm{H}$ NMR $\left(300 \mathrm{MHz}, \mathrm{CDCl}_{3}\right) \delta(\mathrm{ppm}) 6.78(\mathrm{t}, J=2.3 \mathrm{~Hz}, 1 \mathrm{H}), 6.26(\mathrm{t}, J=3.1 \mathrm{~Hz}, 1 \mathrm{H}), 6.22(\mathrm{dd}, J=1.9 \mathrm{and} 3.6 \mathrm{~Hz}, 1 \mathrm{H}), 3.50(\mathrm{~s}, 3 \mathrm{H})$.

${ }^{13} \mathrm{C}$ NMR $\left(100 \mathrm{MHz}, \mathrm{CDCl}_{3}\right) \delta(\mathrm{ppm}) 132.0,126.6,123.8,123.5,123.0,114.0,111.5,107.9,34.4$.

Elemental analysis: calcd (\%) for $\mathrm{C}_{9} \mathrm{H}_{6} \mathrm{BrCl}_{2} \mathrm{NS}$ (311.02): C 34.76, $\mathrm{H}$ 1.94; found: C 34.85, $\mathrm{H} 2.07$.

2-(4-Bromo-2,5-dichlorothiophen-3-yl)-5-butylfuran (2): 2-n-Butylfuran (0.124 g, $1 \mathrm{mmol})$ and 4-bromo-2,5-dichlorothiophene-3-sulfonyl chloride $(0.396 \mathrm{~g}, 1.2 \mathrm{mmol})$ affords 2 in $64 \%(0.227 \mathrm{~g})$ yield.

${ }^{1} \mathrm{H}$ NMR $\left(300 \mathrm{MHz}, \mathrm{CDCl}_{3}\right) \delta(\mathrm{ppm}) 6.70(\mathrm{~d}, J=3.3 \mathrm{~Hz}, 1 \mathrm{H}), 6.12(\mathrm{~d}, J=3.3 \mathrm{~Hz}, 1 \mathrm{H}), 2.69$ (t, $\left.J=7.6 \mathrm{~Hz}, 2 \mathrm{H}\right), 1.69$ (quint., $\left.J=7.6 \mathrm{~Hz}, 2 \mathrm{H}\right), 1.41$ (sext., $J=7.6 \mathrm{~Hz}, 2 \mathrm{H}), 0.95(\mathrm{t}, J=7.6 \mathrm{~Hz}, 3 \mathrm{H})$.

${ }^{13} \mathrm{C} \mathrm{NMR}\left(75 \mathrm{MHz}, \mathrm{CDCl}_{3}\right) \delta(\mathrm{ppm}) 150.4,136.5,122.1,116.9,116.3,105.3,103.5,99.3,23.1,20.8,15.3,6.8$.

Elemental analysis: calcd (\%) for $\mathrm{C}_{12} \mathrm{H}_{11} \mathrm{BrCl}_{2} \mathrm{OS}$ (354.08): C 40.71, $\mathrm{H} 3.13$; found: $\mathrm{C} 40.82, \mathrm{H} 3.36$.

2-(4-Bromo-2,5-dichlorothiophen-3-yl)-3,6-dimethyl-4,5,6,7-tetrahydrobenzofuran (3): Menthofuran (0.150 g, 1 mmol) and 4-bromo-2,5dichlorothiophene-3-sulfonyl chloride (0.396 g, $1.2 \mathrm{mmol})$ affords 3 in $70 \%$ (0.266 g) yield.

${ }^{1} \mathrm{H}$ NMR (400 MHz, $\left.\mathrm{CDCl}_{3}\right) \delta(\mathrm{ppm}) 2.71(\mathrm{dd}, J=5.1$ and $16.4 \mathrm{~Hz}, 1 \mathrm{H}), 2.47-2.33(\mathrm{~m}, 2 \mathrm{H}), 2.27-2.18(\mathrm{~m}, 1 \mathrm{H}), 2.01-1.97(\mathrm{~m}, 1 \mathrm{H}), 1.90(\mathrm{~s}, 3 \mathrm{H}), 1.88-1.82$ $(\mathrm{m}, 1 \mathrm{H}), 1.46-1.35(\mathrm{~m}, 1 \mathrm{H}), 1.1(\mathrm{~d}, J=6.7 \mathrm{~Hz}, 3 \mathrm{H})$.

${ }^{13} \mathrm{C} \mathrm{NMR}\left(100 \mathrm{MHz}, \mathrm{CDCl}_{3}\right) \delta(\mathrm{ppm}) 151.7,138.4,130.6,125.9,123.2,121.0,118.5,112.8,31.5,31.2,29.6,21.6,20.2,9.3$.

Elemental analysis: calcd (\%) for $\mathrm{C}_{14} \mathrm{H}_{13} \mathrm{BrCl}_{2} \mathrm{OS}$ (380.12): C 44.24, $\mathrm{H} 3.45$; found: C 44.37, $\mathrm{H} 3.56$.

1-(5-(4-Bromo-2,5-dichlorothiophen-3-yl)furan-2-yl)propan-2-one (4): 1-(Furan-2-yl)propan-2-one (0.124 g, $1 \quad$ mmol) and 4-bromo-2,5dichlorothiophene-3-sulfonyl chloride (0.396 g, $1.2 \mathrm{mmol})$ affords 4 in $70 \%$ (0.248 g) yield.

${ }^{1} \mathrm{H}$ NMR $\left(400 \mathrm{MHz}, \mathrm{CDCl}_{3}\right) \delta(\mathrm{ppm}) 6.75(\mathrm{~d}, J=3.2 \mathrm{~Hz}, 1 \mathrm{H}), 6.35(\mathrm{~d}, J=3.2 \mathrm{~Hz}, 1 \mathrm{H}), 3.77(\mathrm{~s}, 2 \mathrm{H}), 2.22(\mathrm{~s}, 3 \mathrm{H})$.

${ }^{13} \mathrm{C}$ NMR (100 MHz, $\left.\mathrm{CDCl}_{3}\right) \delta(\mathrm{ppm})$ 203.6, 148.9, 144.8, 128.6, 124.2, 124.1, 112.7, 110.4, 109.7, 43.4, 29.4.

Elemental analysis: calcd (\%) for $\mathrm{C}_{11} \mathrm{H}_{7} \mathrm{BrCl}_{2} \mathrm{O}_{2} \mathrm{~S}$ (354.04): C 37.32, $\mathrm{H}$ 1.99; found: C 37.59, $\mathrm{H} 1.81$.

2-(2,5-Dichlorothiophen-3-yl)benzofuran (5): Benzofuran (0.118 g, 1 mmol) and 4-bromo-2,5-dichlorothiophene-3-sulfonyl chloride (0.396 g, 1.2 $\mathrm{mmol})$ affords 5 in $42 \%(0.113 \mathrm{~g})$ yield.

${ }^{1} \mathrm{H}$ NMR $\left(400 \mathrm{MHz}, \mathrm{CDCl}_{3}\right) \delta(\mathrm{ppm}) 7.61(\mathrm{~d}, J=7.7 \mathrm{~Hz}, 1 \mathrm{H}), 7.5(\mathrm{~d}, J=7.7 \mathrm{~Hz}, 1 \mathrm{H}), 7.35-7.32(\mathrm{~m}, 2 \mathrm{H}), 7.28-7.23(\mathrm{~m}, 2 \mathrm{H})$.

${ }^{13} \mathrm{C} \mathrm{NMR}\left(100 \mathrm{MHz}, \mathrm{CDCl}_{3}\right) \delta(\mathrm{ppm}) 153.8,148.9,128.7,128.3,127.1,125.1,125.0,123.2,122.5,121.3,111.2,104.8$.

Elemental analysis: calcd (\%) for $\mathrm{C}_{12} \mathrm{H}_{6} \mathrm{Cl}_{2} \mathrm{OS}$ (269.14): C 53.55, H 2.25; found: C 53.81, $\mathrm{H} 2.37$.

3-(4-Bromo-2,5-dichlorothiophen-3-yl)benzo[b]thiophene (6): Benzothiophene (0.134 g, 1 mmol) and 4-bromo-2,5-dichlorothiophene-3-sulfonyl chloride $(0.396 \mathrm{~g}, 1.2 \mathrm{mmol})$ affords 6 in $47 \%(0.171 \mathrm{~g})$ yield.

${ }^{1} \mathrm{H}$ NMR (400 MHz, $\left.\mathrm{CDCl}_{3}\right) \delta(\mathrm{ppm}) 7.94-7.91(\mathrm{~m}, 1 \mathrm{H}), 7.55-7.51(\mathrm{~m}, 1 \mathrm{H}), 7.49(\mathrm{~s}, 1 \mathrm{H}), 7.41(\mathrm{q}, J=5.3 \mathrm{~Hz}, 2 \mathrm{H})$.

${ }^{13} \mathrm{C}$ NMR $\left(100 \mathrm{MHz}, \mathrm{CDCl}_{3}\right) \delta(\mathrm{ppm})$ 139.6, 137.4, 133.5, 128.1, 128.1, 128.0, 124.7, 124.4, 123.6, 123.2, $122.8,112.8$.

Elemental analysis: calcd (\%) for $\mathrm{C}_{12} \mathrm{H}_{5} \mathrm{BrCl}_{2} \mathrm{~S}_{2}$ (364.10): C 39.59, H 1.38; found: C 39.79, H 1.12.

2-(2,5-Dichlorothiophen-3-yl)-1-methylpyrrole (7): 1-Methylpyrrole (0.162 g, 2 mmol) and 2,5-dichlorothiophene-3-sulfonyl chloride (0.251 g, 1 mmol) affords 7 in $51 \%(0.118 \mathrm{~g})$ yield.

${ }^{1} \mathrm{H} \mathrm{NMR}\left(400 \mathrm{MHz}, \mathrm{CDCl}_{3}\right) \delta(\mathrm{ppm}) 6.79(\mathrm{~s}, 1 \mathrm{H}), 6.73(\mathrm{dd}, J=1.8$ and $3.1 \mathrm{~Hz}, 1 \mathrm{H}), 6.27(\mathrm{dd}, J=1.8 \mathrm{and} 3.1 \mathrm{~Hz}, 1 \mathrm{H}), 6.22(\mathrm{t}, J=3.1 \mathrm{~Hz}, 1 \mathrm{H}), 3.59(\mathrm{~s}$, $3 \mathrm{H})$.

${ }^{13} \mathrm{C} \mathrm{NMR}\left(100 \mathrm{MHz}, \mathrm{CDCl}_{3}\right) \delta(\mathrm{ppm}) 130.8,127.9,126.1,125.1,124.1,123.5,110.6,107.9,34.8$.

Elemental analysis: calcd (\%) for $\mathrm{C}_{9} \mathrm{H}_{7} \mathrm{Cl}_{2} \mathrm{NS}$ (232.12): C 46.57, H 3.04; found: C 46.71, H 3.32 .

2-(2,5-Dimethylthiophen-3-yl)-1-methylpyrrole (9): 1-Methylpyrrole (0.162 g, 2 mmol) and 2,5-dimethylthiophene-3-sulfonyl chloride (0.211 g, 1 $\mathrm{mmol})$ affords 9 in $36 \%(0.069 \mathrm{~g})$ yield. 
${ }^{1} \mathrm{H}$ NMR $\left(400 \mathrm{MHz}, \mathrm{CDCl}_{3}\right) \delta(\mathrm{ppm}) 6.68(\mathrm{t}, J=2.3 \mathrm{~Hz}, 1 \mathrm{H}), 6.58(\mathrm{~s}, 1 \mathrm{H}), 6.19(\mathrm{t}, J=3.1 \mathrm{~Hz}, 1 \mathrm{H}), 6.07(\mathrm{dd}, J=1.7 \mathrm{and} 3.5 \mathrm{~Hz}, 1 \mathrm{H}), 5.52(\mathrm{~s}, 3 \mathrm{H}), 2.44$ (s, 3H), 2.33 (s, 3H).

${ }^{13} \mathrm{C}$ NMR $\left(100 \mathrm{MHz}, \mathrm{CDCl}_{3}\right) \delta(\mathrm{ppm}) 135.2,134.8,129.8,128.8,127.4,122.0,109.0,107.3,34.5,15.3,14.0$.

Elemental analysis: calcd (\%) for $\mathrm{C}_{11} \mathrm{H}_{13} \mathrm{NS}$ (191.29): C 69.07, H 6.85; found: C 69.33, H 7.05.

Methyl 3-(1-methylpyrrol-2-yl)thiophene-2-carboxylate (10): 1-Methylpyrrole (0.162 g, 2 mmol) and methyl 3-(chlorosulfonyl)thiophene-2carboxylate $(0.241 \mathrm{~g}, 1 \mathrm{mmol})$ affords 9 in $79 \%(0.175 \mathrm{~g})$ yield.

${ }^{1} \mathrm{H}$ NMR $\left(400 \mathrm{MHz}, \mathrm{CDCl}_{3}\right) \delta(\mathrm{ppm}) 7.51(\mathrm{~d}, J=5.1 \mathrm{~Hz}, 1 \mathrm{H}), 7.07(\mathrm{~d}, J=5.1 \mathrm{~Hz}, 1 \mathrm{H}), 6.77(\mathrm{t}, J=2.3 \mathrm{~Hz}, 1 \mathrm{H}), 6.23-6.21(\mathrm{~m}, 2 \mathrm{H}), 3.81(\mathrm{~s}, 3 \mathrm{H}), 3.49(\mathrm{~s}$, $3 \mathrm{H})$.

${ }^{13} \mathrm{C} \mathrm{NMR}\left(100 \mathrm{MHz}, \mathrm{CDCl}_{3}\right) \delta(\mathrm{ppm}) 162.0,139.5,132.2,131.9,130.0,128.4,127.3,123.2,109.6,107.5,52.0,34.4$.

Elemental analysis: calcd (\%) for $\mathrm{C}_{11} \mathrm{H}_{11} \mathrm{NO}_{2} \mathrm{~S}$ (221.27): C 59.71, H 5.01; found: C 59.87, H 5.28.

Methyl 3-(5-butylfuran-2-yl)thiophene-2-carboxylate (11): 2- $n$-Butylfuran (0.124 g, $1 \mathrm{mmol})$ and methyl 3-(chlorosulfonyl)thiophene-2-carboxylate $(0.289 \mathrm{~g}, 1.2 \mathrm{mmol})$ affords $\mathbf{1 1}$ in $66 \%(0.174 \mathrm{~g})$ yield.

${ }^{1} \mathrm{H}$ NMR $\left(400 \mathrm{MHz}, \mathrm{CDCl}_{3}\right) \delta(\mathrm{ppm}) 7.53(\mathrm{~d}, J=5.3 \mathrm{~Hz}, 1 \mathrm{H}), 7.48(\mathrm{~d}, J=3.3 \mathrm{~Hz}, 1 \mathrm{H}), 7.44(\mathrm{~d}, J=5.3 \mathrm{~Hz}, 1 \mathrm{H}), 6.12(\mathrm{~d}, J=3.1 \mathrm{~Hz}, 1 \mathrm{H}), 3.88(\mathrm{~s}, 3 \mathrm{H})$, $2.68(\mathrm{t}, J=7.5 \mathrm{~Hz}, 2 \mathrm{H}), 1.67$ (quint., $J=7.5 \mathrm{~Hz}, 2 \mathrm{H}$ ), 1.41 (sext., $J=7.5 \mathrm{~Hz}, 2 \mathrm{H}), 0.95$ (t, $J=7.5 \mathrm{~Hz}, 3 \mathrm{H}$ ).

${ }^{13} \mathrm{C} \mathrm{NMR}\left(100 \mathrm{MHz}, \mathrm{CDCl}_{3}\right) \delta(\mathrm{ppm}) 162.4,156.8,147.3,137.2,130.1,128.5,122.8,113.9,107.5,52.0,30.2,27.9,22.3,13.9$.

Elemental analysis: calcd (\%) for $\mathrm{C}_{14} \mathrm{H}_{16} \mathrm{O}_{3} \mathrm{~S}$ (264.34): C 63.61, H 6.10; found: C 63.85, H 6.27.

Methyl 3-(5-(2-oxopropyl)furan-2-yl)thiophene-2-carboxylate (12): $\quad 1$-(Furan-2-yl)propan-2-one $\quad(0.124 \quad \mathrm{~g}, \quad 1 \quad$ mmol) $\quad$ and methyl $\quad 3-$ (chlorosulfonyl)thiophene-2-carboxylate $(0.289 \mathrm{~g}, 1.2 \mathrm{mmol})$ affords 12 in $53 \%(0.140 \mathrm{~g})$ yield.

${ }^{1} \mathrm{H}$ NMR $\left(400 \mathrm{MHz}, \mathrm{CDCl}_{3}\right) \delta(\mathrm{ppm}) 7.53-7.47(\mathrm{~m}, 2 \mathrm{H}), 7.46-7.42(\mathrm{~m}, 1 \mathrm{H}), 6.33(\mathrm{~d}, J=2.9 \mathrm{~Hz}, 1 \mathrm{H}), 3.87(\mathrm{~s}, 3 \mathrm{H}), 3.75(\mathrm{~s}, 2 \mathrm{H}), 2.19(\mathrm{~s}, 3 \mathrm{H})$.

${ }^{13} \mathrm{C} \mathrm{NMR}\left(75 \mathrm{MHz}, \mathrm{CDCl}_{3}\right) \delta(\mathrm{ppm}) 203.5,162.2,148.7,148.1,136.4,130.2,128.5,123.9,113.9,110.8,52.0,43.4,29.1$.

Elemental analysis: calcd (\%) for $\mathrm{C}_{13} \mathrm{H}_{12} \mathrm{O}_{4} \mathrm{~S}$ (264.30): C 59.08, H 4.58; found: C 59.26, H 4.81.

Methyl 3-(3,6-dimethyl-4,5,6,7-tetrahydrobenzofuran-2-yl)thiophene-2-carboxylate (13): Menthofuran (0.150 g, 1 mmol) and methyl 3(chlorosulfonyl)thiophene-2-carboxylate $(0.289 \mathrm{~g}, 1.2 \mathrm{mmol})$ affords $\mathbf{1 3}$ in $65 \%(0.189 \mathrm{~g})$ yield.

${ }^{1} \mathrm{H}$ NMR $\left(400 \mathrm{MHz}, \mathrm{CDCl}_{3}\right) \delta(\mathrm{ppm}) 7.46(\mathrm{~d}, J=5.1 \mathrm{~Hz}, 1 \mathrm{H}), 7.17(\mathrm{~d}, J=5.1 \mathrm{~Hz}, 1 \mathrm{H}), 3.84(\mathrm{~s}, 3 \mathrm{H}), 2.71(\mathrm{dd}, J=5.4 \mathrm{and} 16.5 \mathrm{~Hz}, 1 \mathrm{H}), 2.49-2.33(\mathrm{~m}$, $2 \mathrm{H}), 2.27-2.19(\mathrm{~m}, 1 \mathrm{H}), 2.00-1.93(\mathrm{~m}, 1 \mathrm{H}), 1.91(\mathrm{~s}, 3 \mathrm{H}), 1.90-1.82(\mathrm{~m}, 1 \mathrm{H}), 1.46-1.44(\mathrm{~m}, 1 \mathrm{H}), 1.1(\mathrm{~d}, J=6.6 \mathrm{~Hz}, 3 \mathrm{H})$

${ }^{13} \mathrm{C} \mathrm{NMR}\left(75 \mathrm{MHz}, \mathrm{CDCl}_{3}\right) \delta(\mathrm{ppm}) 162.1,150.6,142.0,137.2,130.2,129.9,126.5,119.9,119.2,52.0,31.4,31.3,29.6,21.6,20.2,9.8$

Elemental analysis: calcd (\%) for $\mathrm{C}_{16} \mathrm{H}_{18} \mathrm{O}_{3} \mathrm{~S}$ (290.38): C 66.18, H 6.25; found: C 66.42, H 6.39.

Methyl 3-(benzofuran-2-yl)thiophene-2-carboxylate (14): Benzofuran (0.118 g, $1 \mathrm{mmol})$ and methyl 3-(chlorosulfonyl)thiophene-2-carboxylate (0.289 $\mathrm{g}, 1.2 \mathrm{mmol})$ affords 14 in $62 \%(0.160 \mathrm{~g})$ yield.

${ }^{1} \mathrm{H}$ NMR $\left(400 \mathrm{MHz}, \mathrm{CDCl}_{3}\right) \delta(\mathrm{ppm}) 7.97(\mathrm{~d}, J=1.0 \mathrm{~Hz}, 1 \mathrm{H}), 7.74(\mathrm{~d}, J=5.3 \mathrm{~Hz}, 1 \mathrm{H}), 7.65(\mathrm{dd}, J=1.4 \mathrm{and} 7.7 \mathrm{~Hz}, 1 \mathrm{H}), 7.53(\mathrm{~d}, J=5.4 \mathrm{~Hz}, 1 \mathrm{H}), 7.50-$ $7.49(\mathrm{~m}, 1 \mathrm{H}), 7.33$ (ddd, $J=1.5,7.1$ and $8.2 \mathrm{~Hz}, 1 \mathrm{H}), 7.28-7.22(\mathrm{~m}, 1 \mathrm{H}), 3.93(\mathrm{~s}, 3 \mathrm{H})$.

${ }^{13} \mathrm{C} \mathrm{NMR}\left(75 \mathrm{MHz}, \mathrm{CDCl}_{3}\right) \delta(\mathrm{ppm}) 162.1,154.3,150.2,136.4,130.3,129.4,129.2,126.5,125.2,122.9,121.8,111.0,108.8,52.2$.

Elemental analysis: calcd (\%) for $\mathrm{C}_{14} \mathrm{H}_{10} \mathrm{O}_{3} \mathrm{~S}$ (258.29): C 65.10, H 3.90; found: C 65.31, H 4.17.

1-Methyl-2-(thiophen-2-yl)pyrrole (15): 1-Methylpyrrole (0.162 g, 2 mmol) and thiophene-2-sulfonyl chloride (0.182 g, 1 mmol) affords 15 in $42 \%$ $(0.069 \mathrm{~g})$ yield.

${ }^{1} \mathrm{H}$ NMR $\left(400 \mathrm{MHz}, \mathrm{CDCl}_{3}\right) \delta(\mathrm{ppm}) 7.25(\mathrm{~d}, J=5.1 \mathrm{~Hz}, 1 \mathrm{H}), 7.06(\mathrm{dd}, J=3.8$ and $5.1 \mathrm{~Hz}, 1 \mathrm{H}), 7.02(\mathrm{~d}, J=3.8 \mathrm{~Hz}, 1 \mathrm{H}), 6.72-6.68(\mathrm{~m}, 1 \mathrm{H}), 6.35-6.30$ $(\mathrm{m}, 1 \mathrm{H}), 6.16(\mathrm{t}, J=4.0 \mathrm{~Hz}, 1 \mathrm{H}), 3.71(\mathrm{~s}, 3 \mathrm{H})$.

This is a known compound and the spectral data are identical to those reported in literature. ${ }^{20}$

1-Methyl-2-(5-methylthiophen-2-yl)pyrrole (16): 1-Methylpyrrole (0.162 g, 2 mmol) and 5-methylthiophene-2-sulfonyl chloride (0.182 g, 1 mmol) affords 16 in $46 \%$ (0.082 g) yield.

${ }^{1} \mathrm{H}$ NMR (400 MHz, DMSO-d-6) $\delta(\mathrm{ppm}) 6.90(\mathrm{~s}, 1 \mathrm{H}), 6.82(\mathrm{~s}, 1 \mathrm{H}), 6.81(\mathrm{~s}, 1 \mathrm{H}), 6.14(\mathrm{~s}, 1 \mathrm{H}), 6.01(\mathrm{~s}, 1 \mathrm{H}), 3.66(\mathrm{~s}, 3 \mathrm{H}), 2.43(\mathrm{~s}, 3 \mathrm{H})$.

This is a known compound and the spectral data are identical to those reported in literature. ${ }^{21}$

2-(5-Chlorothiophen-2-yl)-1-methylpyrrole (17): 1-Methylpyrrole (0.162 g, 2 mmol) and 5-chlorothiophene-2-sulfonyl chloride (0.217 g, $1 \mathrm{mmol})$ 
affords 17 in 55\% (0.109 g) yield.

${ }^{1} \mathrm{H}$ NMR $\left(400 \mathrm{MHz}, \mathrm{CDCl}_{3}\right) \delta(\mathrm{ppm}) 7.45(\mathrm{~d}, J=4.1 \mathrm{~Hz}, 1 \mathrm{H}), 7.03(\mathrm{dd}, J=1.6$ and $3.8 \mathrm{~Hz}, 1 \mathrm{H}), 6.93(\mathrm{~d}, J=4.1 \mathrm{~Hz}, 1 \mathrm{H}), 6.83(\mathrm{t}, J=2.3 \mathrm{~Hz}, 1 \mathrm{H}), 6.21$ (dd, $J=2.6$ and $4.1 \mathrm{~Hz}, 1 \mathrm{H}), 3.83$ (s, 3H).

${ }^{13} \mathrm{C}$ NMR (100 MHz, $\left.\mathrm{CDCl}_{3}\right) \delta(\mathrm{ppm})$ 142.1, 138.6, 131.6, 130.2, 128.1, 127.0, 119.0, 108.7, 35.8.

Elemental analysis: calcd (\%) for $\mathrm{C}_{9} \mathrm{H}_{8} \mathrm{ClNS}$ (197.68): C 54.68, H 4.08; found: C 54.89, H 3.83.

2-(5-Bromothiophen-2-yl)-1-methylpyrrole (18): 1-Methylpyrrole $(0.162 \mathrm{~g}, 2 \mathrm{mmol})$ and 5-bromothiophene-2-sulfonyl chloride (0.217 g, $1 \mathrm{mmol})$ affords 18 in $48 \%(0.116 \mathrm{~g})$ yield.

${ }^{1} \mathrm{H}$ NMR $\left(400 \mathrm{MHz}, \mathrm{CDCl}_{3}\right) \delta(\mathrm{ppm}) 7.39(\mathrm{~d}, J=4.0 \mathrm{~Hz}, 1 \mathrm{H}), 7.04(\mathrm{~d}, J=4.0 \mathrm{~Hz}, 1 \mathrm{H}), 7.01(\mathrm{dd}, J=1.9 \mathrm{and} 4.1 \mathrm{~Hz}, 1 \mathrm{H}), 6.81(\mathrm{t}, J=2.3 \mathrm{~Hz}, 1 \mathrm{H}), 6.19$ (dd, $J=2.6$ and $4.1 \mathrm{~Hz}, 1 \mathrm{H}), 3.80(\mathrm{~s}, 3 \mathrm{H})$.

${ }^{13} \mathrm{C}$ NMR $\left(100 \mathrm{MHz}, \mathrm{CDCl}_{3}\right) \delta(\mathrm{ppm}) 145.2,132.3,130.6,130.2,128.1,121.2,119.0,108.7,35.8$.

Elemental analysis: calcd (\%) for $\mathrm{C}_{9} \mathrm{H}_{8} \mathrm{BrNS}$ (242.13): C 44.64, H 3.33; found: C 44.97, H 3.12.

1-Benzyl-2-(5-chlorothiophen-2-yl)pyrrole (19): 1-Benzylpyrrole (0.314 g, $2 \mathrm{mmol})$ and 5-bromothiophene-2-sulfonyl chloride (0.217 g, $1 \mathrm{mmol})$ affords 19 in $62 \%(0.169 \mathrm{~g})$ yield.

${ }^{1} \mathrm{H}$ NMR $\left(400 \mathrm{MHz}, \mathrm{CDCl}_{3}\right) \delta(\mathrm{ppm}) 7.41-7.26(\mathrm{~m}, 5 \mathrm{H}), 7.1(\mathrm{~d}, J=7.9 \mathrm{~Hz}, 2 \mathrm{H}), 6.63(\mathrm{t}, J=2.6 \mathrm{~Hz}, 1 \mathrm{H}), 6.14(\mathrm{t}, J=3.4 \mathrm{~Hz}, 1 \mathrm{H}), 6.10-6.08(\mathrm{~m}, 1 \mathrm{H}), 5.09$ (s, 2H).

${ }^{13} \mathrm{C}$ NMR $\left(100 \mathrm{MHz}, \mathrm{CDCl}_{3}\right) \delta(\mathrm{ppm}) 137.4,136.6,134.2,128.9,128.7,128.6,126.8,126.6,120.7,108.3,106.9,106.7,50.3$.

Elemental analysis: calcd (\%) for $\mathrm{C}_{15} \mathrm{H}_{12} \mathrm{ClNS}$ (273.78): C 65.81, H 4.42; found: $\mathrm{C} 66.07, \mathrm{H} 4.61$.

1,2-Dimethyl-3-(thiophen-2-yl)-indole (21): 1,2-Dimethylindole (0.290 g, $2 \mathrm{mmol})$ and thiophene-2-sulfonyl chloride (0.182 g, $1 \mathrm{mmol})$ affords 21 in $37 \%(0.084 \mathrm{~g})$ yield.

${ }^{1} \mathrm{H}$ NMR $\left(400 \mathrm{MHz}, \mathrm{CDCl}_{3}\right) \delta(\mathrm{ppm}) 7.80(\mathrm{~d}, J=7.8 \mathrm{~Hz}, 1 \mathrm{H}), 7.27-7.35(\mathrm{~m}, 2 \mathrm{H}), 7.22(\mathrm{ddd}, J=0.9,7.1 \mathrm{and} 8.0 \mathrm{~Hz}, 1 \mathrm{H}), 7.11-7.18(\mathrm{~m}, 2 \mathrm{H}), 7.09(\mathrm{dd}, J=$ 0.9 and $3.7 \mathrm{~Hz}, 1 \mathrm{H}), 3.73(\mathrm{~s}, 3 \mathrm{H}), 2.56(\mathrm{~s}, 3 \mathrm{H})$.

This is a known compound and the spectral data are identical to those reported in literature. ${ }^{22}$

3-(1-Methylpyrrol-2-yl)pyridine (22): 1-Methylpyrrole (0.162 g, $2 \mathrm{mmol})$ and pyridine-3-sulfonyl chloride (0.178 g, $1 \mathrm{mmol})$ affords 22 in $31 \%$ (0.049 g) yield.

${ }^{1} \mathrm{H}$ NMR $\left(400 \mathrm{MHz}\right.$, DMSO- $\left.d_{6}\right) \delta(\mathrm{ppm})$ 8.66-8.65 (m, 1H), 8.48-8.47 (m, 1H), 7.87-7.85 (m, 1H), 7.45-7.42 (m, 1H), 6.91-6.90 (m, 1H), 6.28-6.26 (m, $1 \mathrm{H}), 6.11-6.08(\mathrm{~m}, 1 \mathrm{H}), 3.66(\mathrm{~s}, 3 \mathrm{H})$

This is a known compound and the spectral data are identical to those reported in literature. ${ }^{21}$

3-(5-Butylfuran-2-yl)pyridine (23): 2- $n$-Butylfuran $(0.186 \mathrm{~g}, 1.5 \mathrm{mmol})$ and pyridine-3-sulfonyl chloride (0.178 $\mathrm{g}, 1 \mathrm{mmol})$ affords 23 in $12 \%$ (0.024 g) yield.

${ }^{1} \mathrm{H}$ NMR $\left(400 \mathrm{MHz}, \mathrm{CDCl}_{3}\right) \delta(\mathrm{ppm}) 8.88(\mathrm{~m}, 1 \mathrm{H}), 8.49(\mathrm{~m}, 1 \mathrm{H}), 7.85(\mathrm{~d}, J=7.4 \mathrm{~Hz}, 1 \mathrm{H}), 7.25(\mathrm{~m}, 1 \mathrm{H}), 6.62(\mathrm{~d}, J=3.2 \mathrm{~Hz}, 1 \mathrm{H}), 6.07(\mathrm{~d}, J=3.2 \mathrm{~Hz}$, $1 \mathrm{H}), 2.68$ (t, $J=7.4 \mathrm{~Hz}, 2 \mathrm{H}), 1.67$ (quint., $J=7.4 \mathrm{~Hz}, 2 \mathrm{H}), 1.42(\mathrm{~m}, 2 \mathrm{H}), 0.91$ (t, $J=7.4 \mathrm{~Hz}, 3 \mathrm{H}$ ).

This is a known compound and the spectral data are identical to those reported in literature. ${ }^{23}$

Methyl 1,1'-dimethyl-1H,1'H-[2,2'-bipyrrole]-5-carboxylate (24): 1-Methylpyrrole (0.162 g, 2 mmol) and methyl 5-(chlorosulfonyl)-1-methylpyrrole2-carboxylate $(0.238 \mathrm{~g}, 1 \mathrm{mmol})$ affords 24 in $69 \%$ ( $0.151 \mathrm{~g})$ yield.

${ }^{1} \mathrm{H} \mathrm{NMR}\left(400 \mathrm{MHz}, \mathrm{CDCl}_{3}\right) \delta(\mathrm{ppm}) 6.99(\mathrm{~d}, J=2.0 \mathrm{~Hz}, 1 \mathrm{H}), 6.85(\mathrm{~d}, J=1.8 \mathrm{~Hz}, 1 \mathrm{H}), 6.64(\mathrm{t}, J=2.0 \mathrm{~Hz}, 1 \mathrm{H}), 6.13(\mathrm{~d}, J=2.2 \mathrm{~Hz}, 2 \mathrm{H}), 3.95(\mathrm{~s}, 3 \mathrm{H})$, $3.83(\mathrm{~s}, 3 \mathrm{H}), 3.67$ (s, 3H).

${ }^{13} \mathrm{C}$ NMR $\left(100 \mathrm{MHz}, \mathrm{CDCl}_{3}\right) \delta(\mathrm{ppm}) 130.1,128.1,127.6,122.5,120.3,116.8,115.9,107.4,107.2,51.1,36.8,35.0$.

Elemental analysis: calcd (\%) for $\mathrm{C}_{12} \mathrm{H}_{14} \mathrm{~N}_{2} \mathrm{O}_{2}$ (218.26): C 66.04, H 6.47; found: C 66.14, H 6.63.

4-(3-Methylpyrazol-1-yl)benzenesulfonyl chloride (26): To a solution of chlorosulfuric acid $(5 \mathrm{~mL})$ in $\mathrm{CH}_{2} \mathrm{Cl}_{2}(5 \mathrm{~mL})$ a solution of 3-methyl-1phenylpyrazole $(1.58 \mathrm{~g}, 10 \mathrm{mmol})$ in $\mathrm{CH}_{2} \mathrm{Cl}_{2}(5 \mathrm{~mL})$ was slowly added at $0{ }^{\circ} \mathrm{C}$, then the resulting mixture was warm-up to room temperate and stirred during $16 \mathrm{~h}$. Then, the crude mixture was poured in ice and the solid was collected by filtration of affords $\mathbf{2 6}$ in $89 \%$ ( $2.28 \mathrm{~g}$ ) yield.

${ }^{1} \mathrm{H}$ NMR $\left(400 \mathrm{MHz}, \mathrm{CDCl}_{3}\right) \delta(\mathrm{ppm}) 8.01(\mathrm{~d}, J=8.9 \mathrm{~Hz}, 2 \mathrm{H}), 7.91(\mathrm{~s}, 1 \mathrm{H}), 7.84(\mathrm{~d}, J=8.9 \mathrm{~Hz}, 2 \mathrm{H}), 6.32(\mathrm{~s}, 1 \mathrm{H}), 2.35(\mathrm{~s}, 3 \mathrm{H})$.

3-Methyl-1-(4-(1-methylpyrrol-2-yl)phenyl)pyrazole (27): 1-Methylpyrrole (0.162 g, 2 mmol) and 4-(3-methylpyrazol-1-yl)benzenesulfonyl chloride $(0.257 \mathrm{~g}, 1 \mathrm{mmol})$ affords 27 in $72 \%(0.171 \mathrm{~g})$ yield. 
${ }^{1} \mathrm{H}$ NMR $\left(400 \mathrm{MHz}, \mathrm{CDCl}_{3}\right) \delta(\mathrm{ppm}) 7.83(\mathrm{~d}, J=2.5 \mathrm{~Hz}, 1 \mathrm{H}), 7.69(\mathrm{~d}, J=8.6 \mathrm{~Hz}, 2 \mathrm{H}), 7.47(\mathrm{~d}, J=8.6 \mathrm{~Hz}, 2 \mathrm{H}), 6.74(\mathrm{t}, J=2.3 \mathrm{~Hz}, 1 \mathrm{H}), 6.28-6.26(\mathrm{~m}$, $2 \mathrm{H}), 6.23(\mathrm{t}, J=3.0 \mathrm{~Hz}, 1 \mathrm{H}), 3.68(\mathrm{~s}, 3 \mathrm{H}), 2.41(\mathrm{~s}, 3 \mathrm{H})$.

${ }^{13} \mathrm{C}$ NMR $\left(100 \mathrm{MHz}, \mathrm{CDCl}_{3}\right) \delta(\mathrm{ppm}) 150.5,138.7,133.6,131.0,129.4,127.2,123.8,118.7,108.7,107.8,107.6,35.0,13.7$.

Elemental analysis: calcd (\%) for $\mathrm{C}_{15} \mathrm{H}_{15} \mathrm{~N}_{3}$ (237.31): C 75.92, $\mathrm{H}$ 6.37; found: C 76.14, $\mathrm{H}$ 6.59.

3-Methyl-1-(4-(5-methylthiophen-3-yl)phenyl)pyrazole (28): 2-Methylthiophene (0.196 g, 2 mmol) and 4-(3-methylpyrazol-1-yl)benzenesulfonyl chloride $(0.257 \mathrm{~g}, 1 \mathrm{mmol})$ affords 28 in $63 \%(0.160 \mathrm{~g})$ yield.

${ }^{1} \mathrm{H}$ NMR $\left(400 \mathrm{MHz}, \mathrm{CDCl}_{3}\right) \delta(\mathrm{ppm}) 7.82(\mathrm{~d}, J=2.5 \mathrm{~Hz}, 1 \mathrm{H}), 7.66(\mathrm{~d}, J=8.5 \mathrm{~Hz}, 2 \mathrm{H}), 7.61(\mathrm{~d}, J=8.5 \mathrm{~Hz}, 2 \mathrm{H}), 7.21(\mathrm{~d}, J=1.5 \mathrm{~Hz}, 1 \mathrm{H}), 7.07(\mathrm{~s}, 1 \mathrm{H})$, $6.26(\mathrm{~d}, J=2.4 \mathrm{~Hz}, 1 \mathrm{H}), 2.54(\mathrm{~s}, 3 \mathrm{H}), 2.39(\mathrm{~s}, 3 \mathrm{H})$.

${ }^{13} \mathrm{C}$ NMR $\left(100 \mathrm{MHz}, \mathrm{CDCl}_{3}\right) \delta(\mathrm{ppm}) 150.6,141.1,140.8,138.8,134.0,127.4,127.1,124.5,119.1,118.0,107.6,15.5,13.7$.

Elemental analysis: calcd (\%) for $\mathrm{C}_{15} \mathrm{H}_{14} \mathrm{~N}_{2} \mathrm{~S}$ (254.09): C 70.83, H 5.55; found: C 71.12, H 5.41.

2-Ethyl-4-methyl-5-(5-(1-methylpyrrol-2-yl)thiophen-2-yl)thiazole (29): 2-(5-Bromothiophen-2-yl)-1-methylpyrrole (0.242 g, 1 mmol), 2-ethyl-4methylthiazole $(0.191 \mathrm{~g}, 1.5 \mathrm{mmol})$, KOAc $(0.194 \mathrm{~g}, 2 \mathrm{mmol})$ and $\mathrm{PdCl}\left(\mathrm{C}_{3} \mathrm{H}_{5}\right)(\mathrm{dppb})(12.1 \mathrm{mg}, 0.02 \mathrm{mmol})$, were dissolved in DMA (3 mL) under an argon atmosphere. The reaction mixture was stirred at $150{ }^{\circ} \mathrm{C}$ for $16 \mathrm{~h}$. After evaporation of the solvent, the product was purified by silica gel column chromatography to afford $\mathbf{2 9}$ in $78 \%(0.225 \mathrm{~g})$ yield.

${ }^{1} \mathrm{H}$ NMR $\left(400 \mathrm{MHz}, \mathrm{CDCl}_{3}\right) \delta(\mathrm{ppm}) 7.02(\mathrm{~d}, J=3.8 \mathrm{~Hz}, 1 \mathrm{H}), 6.96(\mathrm{~d}, J=3.8 \mathrm{~Hz}, 1 \mathrm{H}), 6.73-6.70(\mathrm{~m}, 1 \mathrm{H}), 6.36(\mathrm{dd}, J=1.4 \mathrm{and} 3.0 \mathrm{~Hz}, 1 \mathrm{H}), 6.17(\mathrm{t}, J=$ $3.0 \mathrm{~Hz}, 1 \mathrm{H}), 3.75$ (s, 3H), 2.98 (q, $J=7.6 \mathrm{~Hz}, 2 \mathrm{H}), 2.57$ (s, 3H), $1.40(\mathrm{t}, J=7.6 \mathrm{~Hz}, 3 \mathrm{H})$.

This is a known compound and the spectral data are identical to those reported in literature. ${ }^{10 \mathrm{i}}$

2-Isobutyl-5-(5-(1-methylpyrrol-2-yl)thiophen-2-yl)thiazole (30): 2-(5-Bromothiophen-2-yl)-1-methylpyrrole (0.242 g, 1 mmol), 2-isobutylthiazole $(0.212 \mathrm{~g}, 1.5 \mathrm{mmol}), \mathrm{KOAc}(0.194 \mathrm{~g}, 2 \mathrm{mmol})$ and $\mathrm{PdCl}\left(\mathrm{C}_{3} \mathrm{H}_{5}\right)(\mathrm{dppb})(12.1 \mathrm{mg}, 0.02 \mathrm{mmol})$, were dissolved in DMA $(3 \mathrm{~mL})$ under an argon atmosphere. The reaction mixture was stirred at $150{ }^{\circ} \mathrm{C}$ for $16 \mathrm{~h}$. After evaporation of the solvent, the product was purified by silica gel column chromatography to afford $\mathbf{3 0}$ in $81 \%$ ( $0.248 \mathrm{~g})$ yield.

${ }^{1} \mathrm{H}$ NMR $\left(400 \mathrm{MHz}, \mathrm{CDCl}_{3}\right) \delta(\mathrm{ppm}) 7.70(\mathrm{~s}, 1 \mathrm{H}), 7.07(\mathrm{~d}, J=3.7 \mathrm{~Hz}, 1 \mathrm{H}), 6.92(\mathrm{~d}, J=3.7 \mathrm{~Hz}, 1 \mathrm{H}), 6.71(\mathrm{dd}, J=1.9$ and $2.7 \mathrm{~Hz}, 1 \mathrm{H}), 6.36(\mathrm{dd}, J=1.9$ and $3.7 \mathrm{~Hz}, 1 \mathrm{H}), 6.17(\mathrm{dd}, J=2.7$ and $3.7 \mathrm{~Hz}, 1 \mathrm{H}), 3.75(\mathrm{~s}, 3 \mathrm{H}), 2.87(\mathrm{~d}, J=7.2 \mathrm{~Hz}, 2 \mathrm{H}), 2.18-2.08(\mathrm{~m}, 1 \mathrm{H}), 1.02(\mathrm{~d}, J=6.7 \mathrm{~Hz}, 6 \mathrm{H})$

${ }^{13} \mathrm{C}$ NMR $\left(100 \mathrm{MHz}, \mathrm{CDCl}_{3}\right) \delta(\mathrm{ppm})$ 169.1, 137.5, 135.0, 132.1, 131.7, 126.6, 125.6, 125.1, 124.6, 110.4, 108.2, 42.4, 35.4, 29.8, 22.3.

Elemental analysis: calcd (\%) for $\mathrm{C}_{16} \mathrm{H}_{18} \mathrm{~N}_{2} \mathrm{~S}_{2}$ (302.45): C 63.54, H 6.00; found: C 63.82, H 5.79.

\section{Notes and references}

${ }^{a}$ Institut des Sciences Chimiques de Rennes, UMR 6226 CNRS-Université de Rennes 1 " Organométalliques, Matériaux et Catalyse", Campus de Beaulieu, 35042 Rennes, France

${ }^{b}$ Laboratoire de synthèse des molecules à intérêt biologique Université Mentouri-Constantine, 25000 Constantine, Algeria

$\dagger$ Electronic Supplementary Information (ESI) available: [details of any supplementary information available should be included here]. See DOI: $10.1039 / \mathrm{b} 000000 \mathrm{x} /$

1. L. M. Espinoza-Fonseca, Theor. Biol. Med. Model. , 2005, 2, 38-38.

2. K. Kikuchi, S. Hibi, H. Yoshimura, K. Tai, T. Hida, N. Tokuhara, T. Yamauchi and M. Nagai, Bioorg. Med. Chem. Lett., 2000, 10, 619-622.

3. H. Yoshimura, K. Kikuchi, S. Hibi, K. Tagami, T. Satoh, T. Yamauchi, A. Ishibahi, K. Tai, T. Hida, N. Tokuhara and M. Nagai, J. Med. Chem., 2000, 43, 2929-2937.

4. a) D. Zhao, J. You and C. Hu, Chem. Eur. J., 2011, 17, 5466-5492; b) B. Alcaide, P. Almendros, J. M. Alonso and I. Fernández, J. Org. Chem., 2013, 78, 6688-6701; c) B. Alcaide, P. Almendros and C. Lazaro-Milla, Chem. Commun., 2015, 51, 6992-6995.

5. a) J. J. Li and G. W. Gribble, Palladium in Heterocyclic Chemistry, Pergamon, Amsterdam, 2000; b) L. Ackermann, Modern arylation methods, Wiley, 2009; c) C. C. C. Johansson Seechurn, M. O. Kitching, T. J. Colacot and V. Snieckus, Angew. Chem. Int. Ed., 2012, 51, 5062-5085.

6. a) Y. Yang, A. B. Hoernfeldt and S. Gronowitz, Synthesis, 1989, 130-131; b) K. Yui, Y. Aso, T. Otsubo and F. Ogura, Bull. Chem. Soc. Jpn., 1989, 62, 1539-1546; c) R. Rossi, A. Carpita, M. Ciofalo and V. Lippolis, Tetrahedron, 1991, 47, 8443-8460; d) G. Palmisano and M. Santagnostino, Synlett, 1993, 771-773; e) R. L. Hudkins, J. L. Diebold and F. D. Marsh, J. Org. Chem., 1995, 60, 6218-6220; f) T. Bach and L. Krueger, Synlett, 1998, 1185-1186; g) P. R. L. Malenfant, L. Groenendaal and J. M. J. Frechet, J. Am. Chem. Soc., 1998, 120, 10990-10991; h) F. Odobel, S. Suresh, E. Blart, Y. Nicolas, J.-P. Quintard, P. Janvier, J.-Y. Le Questel, B. Illien, D. Rondeau, P. Richomme, T. Haupl, S. Wallin and L. Hammarstrom, Chem. Eur. J., 2002, 8, 3027-3046; i) H. V. Huynh, X. He and T. Baumgartner, Chem. Commun., 2013, 49, 4899-4901; j) J.-F. Jheng, Y.-Y. Lai, J.-S. Wu, Y.-H. Chao, C.-L. Wang and C.-S. Hsu, Adv. Mater. , 2013, 25, 2445-2451; k) Q. Wu, S. Ren, M. Wang, X. Qiao, H. Li, X. Gao, X. Yang and D. Zhu, Adv. Funct. Mater., 2013, 23, 2277-2284; 1) J. Zhou, S. Xie, E. F. Amond and M. L. Becker, Macromolecules, 2013, 46, 3391-3394; m) X. Liu, Y. Sun, B. B. Y. Hsu, A. Lorbach, L. Qi, A. J. Heeger and G. C. Bazan, J. Am. Chem. Soc., 2014, 136, 5697-5708; n) R. L. Uy, L. Yan, W. Li and W. You, Macromolecules, 2014, 47, 2289-2295; o) R. Yeh-Yung Lin, F.-L. Wu, C.-H. Chang, H.-H. Chou, T.-M. Chuang, T.-C. Chu, C.-Y. Hsu, P.-W. Chen, K.-C. Ho, Y.-H. Lo and J. T. Lin, J. Mater. Chem. A, 2014, 2, 3092-3101; p) M. Nakano, I. Osaka and K. Takimiya, Macromolecules, 2015 , 48, 576-584.

7. a) J. M. Clough, I. S. Mann and D. A. Widdowson, Tetrahedron Lett., 1987, 28, 2645-2648; b) D. Prim, J. Giner Planas, A. Auffrant, F. Rose-Munch, E. Rose and J. Vaissermann, J. Organomet. Chem., 2003, 688, 273-279; c) D. H. Boschelli, B. Wu, A. C. B. Sosa, H. Durutlic, J. J. Chen, Y. Wang, J. M. Golas, J. Lucas and F. Boschelli, J. Med. Chem., 2005, 48, 3891-3902; d) L. Ilies, H. Tsuji, Y. Sato and E. Nakamura, J. Am. Chem. Soc., 2008, 130, 4240-4241; e) P. van Rijn, T. J. Savenije, M. C. A. Stuart and J. H. van Esch, Chem. Commun., 2009, 2163-2165. 
8. a) T. Otsubo, Y. Kono, N. Hozo, H. Miyamoto, Y. Aso, F. Ogura, T. Tanaka and M. Sawada, Bull. Chem. Soc. Jpn., 1993, 66, 2033-2041; b) P. G. Ciattini, E. Morera and G. Ortar, Tetrahedron Lett., 1994, 35, 2405-2408; c) K. Tanaka, H. Suzuki and H. Osuga, Tetrahedron Lett., 1997, 38, 457460; d) M. Alvarez, D. Fernandez and J. A. Joule, Synthesis, 1999, 615-620; e) L. San Miguel, W. W. Porter, III and A. J. Matzger, Org. Lett., 2007, 9, 1005-1008; f) L.-Y. Yang, C.-F. Chang, Y.-C. Huang, Y.-J. Lee, C.-C. Hu and T.-H. Tseng, Synthesis, 2009, $1175-1179$.

9. a) E. M. Beccalli, G. Broggini, M. Martinelli and S. Sottocornola, Chem. Rev., 2007, 107, 5318-5365; b) S. Pascual, P. de Mendoza and A. M. Echavarren, Org. Biomol. Chem., 2007, 5, 2727-2734; c) F. Bellina, S. Cauteruccio and R. Rossi, Curr. Org. Chem., 2008, 12, 774-790; d) F. Kakiuchi and T. Kochi, Synthesis, 2008, 3013-3039; e) B.-J. Li, S.-D. Yang and Z.-J. Shi, Synlett, 2008, 949-957; f) L. Ackermann, R. Vicente and A. R. Kapdi, Angew. Chem. Int. Ed., 2009, 48, 9792-9826; g) X. Chen, K. M. Engle, D.-H. Wang and J.-Q. Yu, Angew. Chem. Int. Ed., 2009, 48, 50945115; h) T. W. Lyons and M. S. Sanford, Chem. Rev., 2010, 110, 1147-1169; i) T. Satoh and M. Miura, Synthesis, 2010, 3395-3409; j) S. H. Cho, J. Y. Kim, J. Kwak and S. Chang, Chem. Soc. Rev., 2011, 40, 5068-5083; k) N. Kuhl, M. N. Hopkinson, J. Wencel-Delord and F. Glorius, Angew. Chem. Int. Ed., 2012, 51, 10236-10254; 1) B.-J. Li and Z.-J. Shi, Chem. Soc. Rev., 2012, 41, 5588-5598; m) D.-G. Yu, B.-J. Li and Z.-J. Shi, Tetrahedron, 2012, 68, 5130-5136; n) M. He, J.-F. Soulé and H. Doucet, ChemCatChem, 2014, 6, 1824-1859; o) R. Rossi, F. Bellina, M. Lessi and C. Manzini, Adv. Synth. Catal., 2014, 356, 17-117; p) K. Yuan, J.-F. Soulé and H. Doucet, ACS Catal., $2015,5,978-991$.

10. a) S. Pivsa-Art, T. Satoh, Y. Kawamura, M. Miura and M. Nomura, Bull. Chem. Soc. Jpn., 1998, 71, 467-473; b) N. Masuda, S. Tanba, A. Sugie, D. Monguchi, N. Koumura, K. Hara and A. Mori, Org. Lett., 2009, 11, 2297-2300; c) S. Tanba, A. Sugie, N. Masuda, D. Monguchi, N. Koumura, K. Hara and A. Mori, Heterocycles, 2010, 82, 505-529; d) D. Takeda, M. Yamashita, K. Hirano, T. Satoh and M. Miura, Chem. Lett., 2011, 40, 10151017; e) H. Y. Fu, L. Zhao, C. Bruneau and H. Doucet, Synlett, 2012, 23, 2077-2082; f) J. Zhang, D.-Y. Kang, S. Barlow and S. R. Marder, J. Mater. Chem., 2012, 22, 21392-21394; g) J. Laroche, K. Beydoun, V. Guerchais and H. Doucet, Catal. Sci. Technol., 2013, 3, 2072-2080; h) S. Preciado, L. Mendive-Tapia, F. Albericio and R. Lavilla, J. Org. Chem., 2013, 78, 8129-8135; i) F. Belkessam, A. Mohand, J.-F. Soulé, A. Elias and H. Doucet, Beilstein J. Org. Chem., 2014, 10, 2912-2919; j) A. D. Hendsbee, C. M. Macaulay and G. C. Welch, Dyes Pigm., 2014, 102, 204-209; k) Y. Li, J. Wang, M. Huang, Z. Wang, Y. Wu and Y. Wu, J. Org. Chem., 2014, 79, 2890-2897; 1) B. Koszarna, R. Matczak, M. Krzeszewski, O. Vakuliuk, J. Klajn, M. Tasior, J. T. Nowicki and D. T. Gryko, Tetrahedron, 2014, 70, 225-231.

11. a) A. K. Yadav, H. Ila and H. Junjappa, Eur. J. Org. Chem., 2010, 338-344; b) E. Coya, N. Sotomayor and E. Lete, Adv. Synth. Catal., 2014, 356, 1853-1865; c) T. T. H. Luong, J.-D. Brion, M. Alami and S. Messaoudi, J. Org. Chem., 2015, 80, 751-761; d) Y. L. Choi, H. Lee, B. T. Kim, K. Choi and J.-N. Heo, Adv. Synth. Catal., 2010, 352, 2041-2049.

12. a) N. I. Abdo, A. A. El-Shehawy, A. A. El-Barbary and J.-S. Lee, Eur. J. Org. Chem., 2012, 2012, 5540-5551; b) B. Liu, Z. Wang, N. Wu, M. Li, J. You and J. Lan, Chem. Eur. J., 2012, 18, 1599-1603; c) M. J. Robb, D. Montarnal, N. D. Eisenmenger, S.-Y. Ku, M. L. Chabinyc and C. J. Hawker, Macromolecules, 2013, 46, 6431-6438; d) C. Moussalem, O. Segut, F. Gohier, M. Allain and P. Frere, ACS Sustainable Chem. Eng., 2014, 2, 10431048; e) M. Wakioka, N. Ichihara, Y. Kitano and F. Ozawa, Macromolecules, 2014, 47, 626-631; f) P.-O. Morin, T. Bura, B. Sun, S. I. Gorelsky, Y. Li and M. Leclerc, ACS Macro Lett., 2015, 4, 21-24; g) J. G. Weis and T. M. Swager, ACS Macro Lett., 2015, 4, $138-142$.

13. a) K. Yuan and H. Doucet, Chem. Sci., 2014, 5, 392-396; b) A. Beladhria, K. Yuan, H. Ben Ammar, J.-F. Soulé, R. Ben Salem and H. Doucet, Synthesis, 2014, 46, 2515-2523; c) R. Jin, K. Yuan, E. Chatelain, J.-F. Soulé and H. Doucet, Adv. Synth. Catal., 2014, 356, $3831-3841$.

14. a) N. S. Johary and L. N. Owen, J. Chem. Soc., 1955, 1307-1311; b) A. Barco, S. Benetti, G. P. Pollini and R. Taddia, Synthesis, 1974, 877-878; c) S. Fujita, Synthesis, 1982, 423-424; d) T. Kataoka, T. Iwama, T. Setta and A. Takagi, Synthesis, 1998, 423-426; e) G. Blotny, Tetrahedron Lett., 2003, 44, 1499-1501.

15. a) M. Romero, Y. Harrak, J. Basset, L. Ginet, P. Constans and M. D. Pujol, Tetrahedron, 2006, 62, 9010-9016; b) S. Bensaid and H. Doucet, Tetrahedron, 2012, 68, 7655-7662.

16. H. M. Gilow and D. E. Burton, J. Org. Chem., 1981, 46, 2221-2225.

17. a) X. Sun, J. Qiu, S. A. Strong, L. S. Green, J. W. F. Wasley, J. P. Blonder, D. B. Colagiovanni, S. C. Mutka, A. M. Stout, J. P. Richards and G. J. Rosenthal, Bioorg. Med. Chem. Lett., 2011, 21, 5849-5853; b) X. Sun, J. W. F. Wasley, J. Qiu, J. P. Blonder, A. M. Stout, L. S. Green, S. A. Strong, D. B. Colagiovanni, J. P. Richards, S. C. Mutka, L. Chun and G. J. Rosenthal, ACS Med. Chem. Lett., 2011, 2, $402-406$.

18. X. Zhao and V. M. Dong, Angew. Chem. Int. Ed., 2011, 50, 932-934.

19. T. Cantat, E. Génin, C. Giroud, G. Meyer and A. Jutand, J. Organomet. Chem., 2003, 687, 365-376.

20. S. Lotz, C. Crause, A. J. Olivier, D. C. Liles, H. Gorls, M. Landman and D. I. Bezuidenhout, Dalton Trans., 2009, 697-710.

21. F. Bilodeau, M.-C. Brochu, N. Guimond, K. H. Thesen and P. Forgione, J. Org. Chem., 2010, 75, 1550-1560.

22. T. Tsuchimoto, M. Iwabuchi, Y. Nagase, K. Oki and H. Takahashi, Angew. Chem. Int. Ed., 2011, 50, $1375-1379$.

23. J. J. Dong, J. Roger, F. Pozgan and H. Doucet, Green Chem., 2009, 11, 1832-1846. 